\title{
The leading hadronic contribution to $\gamma-Z$ mixing
}

\author{
Vera Gülpers ${ }^{* 1}$, Harvey Meyer ${ }^{1,2}$, Georg von Hippel ${ }^{1}$, Hartmut Wittig ${ }^{1,2}$ \\ ${ }^{1}$ PRISMA Cluster of Excellence, Institut für Kernphysik, Johannes Gutenberg Universität Mainz, \\ 55099 Mainz, Germany \\ ${ }^{2}$ Helmholtz Institute Mainz, Johannes Gutenberg Universität Mainz, 55099 Mainz, Germany \\ E-mail: guelpers@kph.uni-mainz.de
}

\begin{abstract}
We study the leading hadronic contribution to $\gamma-Z$ mixing, which determines the leading order hadronic contribution to the running of the electroweak mixing angle $\theta_{W}$. The required vacuum polarization function $\Pi^{\gamma Z}$ is calculated from the appropriate vector correlation functions in a mixed time-momentum representation. We explicitly calculate the connected and the disconnected contributions to such vector correlators using $N_{f}=2$ dynamical flavors of nonperturbatively $O(a)$-improved Wilson fermions.
\end{abstract}

The 33rd International Symposium on Lattice Field Theory

14 -18 July 2015

Kobe International Conference Center, Kobe, Japan*

${ }^{*}$ Speaker. 


\section{Introduction}

The electroweak mixing angle $\sin ^{2} \theta_{W}$ is a fundamental parameter in the Standard Model of particle physics, which is related to the unification of the electromagnetic and the weak interactions, and is determined by the mixing of the $Z$ boson and the photon. Quantum corrections cause it to run with the momentum scale $Q^{2}$. The hadronic contribution to the running of the Weinberg angle is given in terms of a hadronic vacuum polarization $\Pi^{\gamma Z}$ with

$$
\Pi_{\mu v}^{\gamma Z}(Q) \equiv \int \mathrm{d}^{4} x e^{i Q \cdot x}\left\langle\left. j_{\mu}^{Z}\right|_{\text {vector }}(x) j_{v}^{\gamma}(0)\right\rangle=\left(Q_{\mu} Q_{v}-\delta_{\mu v} Q^{2}\right) \Pi^{\gamma Z}\left(Q^{2}\right),
$$

with the electromagnetic photon current $j_{\mu}^{\gamma}$ and the $Z$ current $j_{\mu}^{Z}$. The corresponding currents are given by [1]

$$
\begin{aligned}
j_{\mu}^{\gamma} & =\frac{2}{3} \bar{u} \gamma_{\mu} u-\frac{1}{3} \bar{d} \gamma_{\mu} d-\frac{1}{3} \bar{s} \gamma_{\mu} s+\frac{2}{3} \bar{c} \gamma_{\mu} c \\
\left.j_{\mu}^{Z}\right|_{\text {vector }} & =\left.j_{\mu}^{3}\right|_{\text {vector }}-\sin ^{2} \theta_{W} j_{\mu}^{\gamma} \\
\left.j_{\mu}^{3}\right|_{\text {vector }} & =\frac{1}{4} \bar{u} \gamma_{\mu} u-\frac{1}{4} \bar{d} \gamma_{\mu} d-\frac{1}{4} \bar{s} \gamma_{\mu} s+\frac{1}{4} \bar{c} \gamma_{\mu} c .
\end{aligned}
$$

Since QCD is invariant under charge conjugation, only the vector part of the $Z$ current has to be considered.

\section{The correlator for $\gamma-Z$ mixing}

To calculate the vacuum polarization $\Pi^{\gamma Z}$, we will use the appropriate zero-momentum correlator in mixed time-momentum representation,

$$
G^{\gamma Z}\left(x_{0}\right)=-\frac{1}{3} \sum_{k} \int \mathrm{d}^{3} x\left\langle\left. j_{k}^{Z}(x)\right|_{\text {vector }} j_{k}^{\gamma}(0)\right\rangle \quad \text { with } \quad k=1,2,3 .
$$

The subtracted $\gamma Z$ vacuum polarization is obtained from the correlator $G^{\gamma Z}\left(x_{0}\right)$ by integrating over the remaining time direction,

$$
\Pi_{R}^{\gamma Z}\left(Q^{2}\right) \equiv \Pi^{\gamma Z}\left(Q^{2}\right)-\Pi^{\gamma Z}(0)=\int_{0}^{\infty} \mathrm{d} x_{0} G^{\gamma Z}\left(x_{0}\right)\left[x_{0}^{2}-\frac{4}{Q^{2}} \sin ^{2}\left(\frac{1}{2} Q x_{0}\right)\right] .
$$

This method has originally been developed for the calculation of the hadronic contribution to the anomalous magnetic moment of the muon [2,3,4]. The hadronic contribution to the running of the Weinberg angle is directly proportional to the subtracted vacuum polarization [5]

$$
\Delta_{\mathrm{had}} \sin ^{2} \theta_{W}\left(Q^{2}\right)=\frac{e^{2}}{\sin ^{2} \theta_{W}} \Pi_{R}^{\gamma Z}\left(Q^{2}\right) .
$$

Writing down the Wick contractions for the correlator (2.1) one finds connected and disconnected contributions as depicted in figure 1 . The connected correlator for four quark flavors is given by

$$
G_{\mathrm{con}}^{\gamma Z}\left(x_{0}\right)=\left(\frac{1}{4}-\frac{5}{9} \sin ^{2} \theta_{W}\right) G^{\ell}\left(x_{0}\right)+\left(\frac{1}{12}-\frac{1}{9} \sin ^{2} \theta_{W}\right) G^{S}\left(x_{0}\right)+\left(\frac{1}{6}-\frac{4}{9} \sin ^{2} \theta_{W}\right) G^{c}\left(x_{0}\right) .
$$

The corresponding single flavor correlators $G^{f}\left(x_{0}\right)$ can be expressed as

$$
G^{f}\left(x_{0}\right) \equiv \frac{1}{3} \sum_{k} \sum_{\vec{x}}\left\langle\operatorname{Tr}\left[D_{f}^{-1}(x, 0) \gamma_{k} D_{f}^{-1}(0, x) \gamma_{k}\right]\right\rangle_{G}
$$

in terms of quark propagators. The disconnected Wick contractions will be discussed in section 4 . 

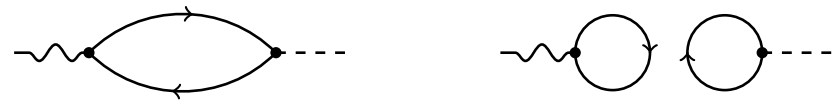

Figure 1: The connected and the disconnected contribution to $\gamma-Z$ mixing.

We also tested a different strategy to only calculate a correlation function of the form

$$
G^{3 \gamma}\left(x_{0}\right)=-\frac{1}{3} \sum_{k} \int \mathrm{d}^{3} x\left\langle\left(\left.j_{k}^{3}(x)\right|_{\text {vector }}-\frac{9}{20} j_{k}^{\gamma}(x)\right) j_{k}^{\gamma}(0)\right\rangle
$$

on the lattice and take the remaining required vacuum polarization $\propto \Pi^{\gamma \gamma}$ from dispersion relation and phenomenology. The advantage of this approach is that the correlation function (2.6) does not include a light connected piece. However, in this study we resort to calculate the complete correlator (2.1) using lattice QCD.

\section{Results for the connected contribution}

For the analysis of the connected contribution we follow the steps of [6], where the vector correlator in time momentum representation has been used to determine the leading hadronic contribution to the anomalous magnetic moment of the muon.
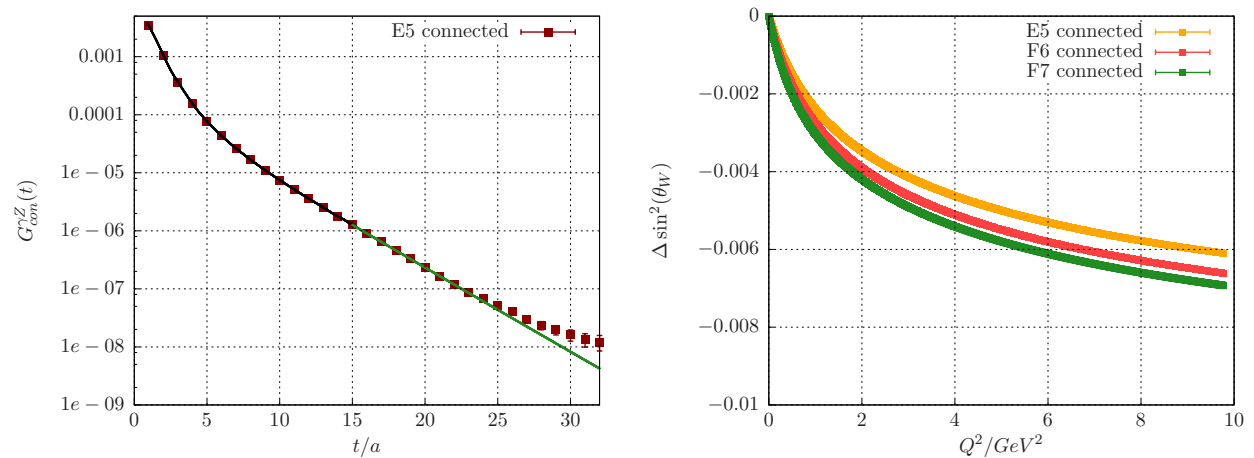

Figure 2: The plot on the left-hand side shows the result for the correlator $G_{\mathrm{con}}^{\gamma Z}\left(x_{0}\right)$ plotted against $t$ for the E5 ensemble (cf. table 1 for details of the ensembles). The green curve indicates a fit to our data with a fit range of $t \in[15,20]$. The connected hadronic contribution to the running of the Weinberg angle is plotted against the momentum $Q^{2}$ for all three ensembles on the right.

The connected correlator $G_{\mathrm{con}}^{\gamma Z}\left(x_{0}\right)$ for the E5 ensemble (cf. table 1) is plotted on the left-hand side of figure 2 against the source-sink separation $t$. The connected correlator has been calculated with a conserved vector current at the sink and a local current at the source. The required multiplicative renormalization $Z_{v}$ is taken from [7]. To obtain the subtracted vacuum polarization $\Pi_{R}^{\gamma Z}\left(Q^{2}\right)$, one has to integrate the correlator over Euclidean time. For this purpose we interpolate the correlator with cubic splines for $t \leq 15$ as indicated by the black curve in figure 2. For large Euclidean times $t>15$ we fit the correlator for every quark flavor separately to a function $\propto \exp \left(-m_{f} t\right)$ (shown by the green line in figure 2) and use the result of this fit to integrate up to $t \rightarrow \infty$. From the subtracted vacuum polarization we obtain the hadronic contribution to the running of the Weinberg angle $\Delta_{\text {had }} \sin ^{2} \theta_{W}\left(Q^{2}\right)$ according to equation (2.3). Here we use the result for the fine structure constant $\alpha^{-1}$ from [8] and the tree-level Weinberg angle in the $\overline{\mathrm{MS}}$ scheme $\sin ^{2} \theta_{W}(0)=0.23871(9)$ 


\begin{tabular}{|ccccccc|}
\hline$\beta$ & $a[\mathrm{fm}]$ & lattice & $m_{\pi}[\mathrm{MeV}]$ & $m_{\pi} L$ & Label & $N_{\text {cnfg }}$ \\
\hline \hline 5.3 & 0.063 & $64 \times 32^{3}$ & 455 & 4.7 & E5 & 1000 \\
5.3 & 0.063 & $96 \times 48^{3}$ & 325 & 5.0 & F6 & 300 \\
5.3 & 0.063 & $96 \times 48^{3}$ & 280 & 4.3 & F7 & 250 \\
\hline
\end{tabular}

Table 1: Overview of the CLS ensembles that have been used for the calculation of the hadronic contribution to the running of the Weinberg angle.

from [9]. Our results for $\Delta_{\text {had }} \sin ^{2} \theta_{W}\left(Q^{2}\right)$ are shown in the right-hand side of figure 2 for the three gauge ensembles listed in table 1 . We observe a clear trend in $\Delta_{\text {had }} \sin ^{2} \theta_{W}\left(Q^{2}\right)$ as the pion mass is lowered.

\section{The disconnected contribution}

As already discussed, the correlator $G^{\gamma Z}\left(x_{0}\right)$ receives a disconnected contribution. For three quark flavors the disconnected Wick contractions are given by

$$
G_{\mathrm{disc}}^{\gamma Z}\left(x_{0}\right) \equiv-\frac{1}{3} \sum_{k} \int \mathrm{d}^{3} x\left\langle\left. j_{k}^{Z}(x)\right|_{\mathrm{vector}} j_{k}^{\gamma}(0)\right\rangle_{\mathrm{disc}}=\sin ^{2} \theta_{W} \frac{1}{9} G_{\mathrm{disc}}^{(\ell+A s),(\ell-s)}\left(x_{0}\right) .
$$

The disconnected correlator $G_{\mathrm{disc}}^{(\ell+A s),(\ell-s)}$ is given as

$$
\begin{aligned}
G_{\mathrm{disc}}^{(\ell+A s),(\ell-s)}\left(x_{0}-y_{0}\right)=\frac{1}{3} \sum_{k} \frac{1}{L^{3}}\left\langle\left(\sum_{\vec{x}}\right.\right. & \left.\operatorname{Tr}\left[\gamma_{k} D_{\ell}^{-1}(x, x)+A \gamma_{k} D_{s}^{-1}(x, x)\right]\right) \\
& \left.\times\left(\sum_{\vec{y}} \operatorname{Tr}\left[\gamma_{k} D_{\ell}^{-1}(y, y)-\gamma_{k} D_{s}^{-1}(y, y)\right]\right)\right\rangle
\end{aligned}
$$

in terms of quark propagators with $A=\frac{3}{4 \sin ^{2} \theta_{W}}-1$. In the second row of equation (4.2) one can see that at the source $y$ only the difference of light and strange propagators is needed. Since we calculated the required disconnected quark loops with stochastic sources, we can calculate both - light and strange quark loops - with the same sources, such that the stochastic noise is largely canceled at the source $y$. This noise cancellation has already been used in our calculation of the disconnected contribution to $a_{\mu}$ [10], where one obtains a cancellation at the source and the sink.
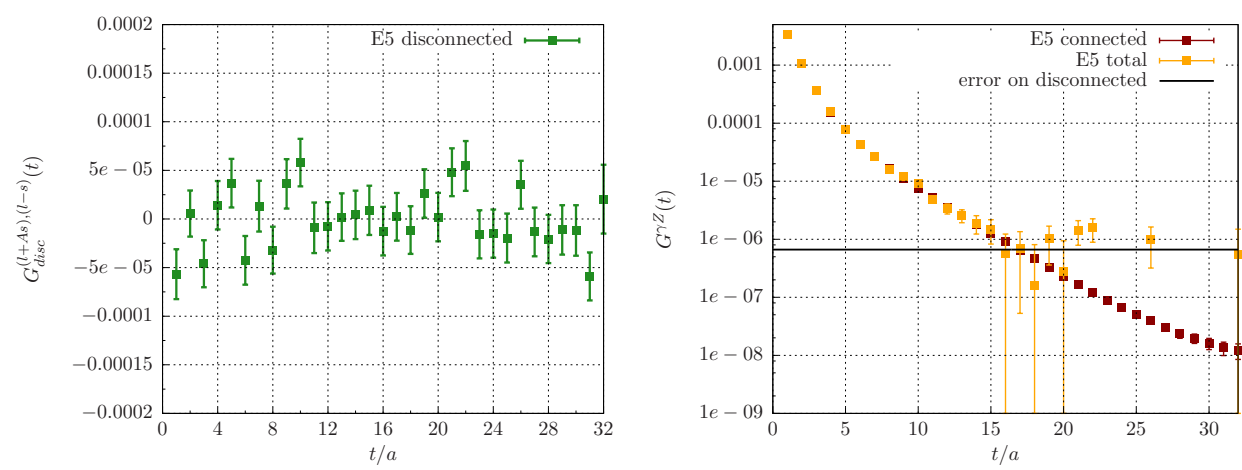

Figure 3: The disconnected correlator is plotted on the left-hand side. The right-hand side shows the connected correlator (red) and the total correlator (yellow). The black line indicates the level of the error on the disconnected contribution. 
The left-hand side of figure 3 shows the disconnected correlator $G_{\text {disc }}^{(\ell+A s),(\ell-s)}$. The disconnected quark loops have been calculated with three stochastic sources per timeslice and a generalized hopping parameter expansion [11]. As discussed above, light and strange propagators are estimated with the same stochastic sources. We find that the disconnected correlator is consistent with zero within the statistical errors. However, we have to add the disconnected contribution to the connected one to obtain the total correlator $G^{\gamma Z}$. This is shown by the yellow points in the right-hand side of figure 3. The black line indicates the level of the error on the disconnected contribution. For $t \gtrsim 16$ the total correlator is dominated by the noise from the disconnected contribution.

\section{The correlator for large Euclidean times}

Since we find the disconnected correlator to be consistent with zero within statistical errors, we have to resort to giving an upper limit for the maximum possible disconnected contribution to $\Delta_{\text {had }} \sin ^{2} \theta_{W}\left(Q^{2}\right)$. For this purpose we investigate the behavior of the correlator for large Euclidean times using the same arguments as in $[3,10]$ for for the hadronic vacuum polarization contribution $a_{\mu}^{\mathrm{HVP}}$.

Since the lowest possible isovector state consists of two pions and the lowest isoscalar state of three pions, the isovector channel opens at energies of $2 m_{\pi}$ whereas the isoscalar channel opens only at $3 m_{\pi}$. Thus one expects for large Euclidean times that the correlator is dominated by the isovector part, which is given by

$$
G^{\gamma Z}(t) \longrightarrow\left(\frac{1}{2}-\sin ^{2} \theta_{W}\right) G^{\rho \rho}(t) \quad \text { for } \quad t \rightarrow \infty
$$

with the $\rho$-correlator $G^{\rho \rho} \equiv \frac{1}{2} G^{\ell}$. On the other hand, we can rewrite the expression for the total correlator $G^{\gamma Z}(t)$ (neglecting charm contributions) by dividing by the $\rho$-correlator and rearranging the terms,

$$
\frac{1}{9} \sin ^{2} \theta_{W} \frac{G_{\mathrm{dis}}^{(\ell+A s),(\ell-s)}(t)}{G^{\rho \rho}(t)}=\frac{G^{\gamma Z}(t)-\left(\frac{1}{2}-\sin ^{2} \theta_{W}\right) G^{\rho \rho}(t)}{G^{\rho \rho}(t)}+\frac{1}{9} \sin ^{2} \theta_{W}-\left(\frac{1}{6}-\frac{2}{9} \sin ^{2} \theta_{W}\right) \frac{G^{s}(t)}{G^{\ell}(t)} .
$$

For large Euclidean times, the first term on the right-hand side of (5.2) vanishes due to equation (5.1). Additionally, the term $\propto G^{s} / G^{\ell}$ is exponentially suppressed and vanishes for $t \rightarrow \infty$. Hence, we find

$$
G_{\mathrm{disc}}^{(\ell+A s),(\ell-s)}(t) \longrightarrow \frac{1}{2} G^{\ell}(t)=G^{\rho \rho}(t) \quad \text { for } \quad t \rightarrow \infty
$$

for the large time behavior of the disconnected correlator.

Figure 4 shows the ratio of the disconnected correlator and the $\rho$-correlator. This ratio is expected to be 1 for $t \rightarrow \infty$ according to equation (5.3). The green line on the left-hand side indicates the asymptotic value. Clearly, the ratio can be distinguished from the asymptotic value 1 up to $t \approx 8 a$. We are interested in the scenario where the disconnected diagram contributes as much as possible while still agreeing with our data. If we assume that the ratio rises monotonically from $\approx 0$ to its asymptotic value, the maximum possible contribution is shown by the blue curve on the right-hand side of figure 4. 

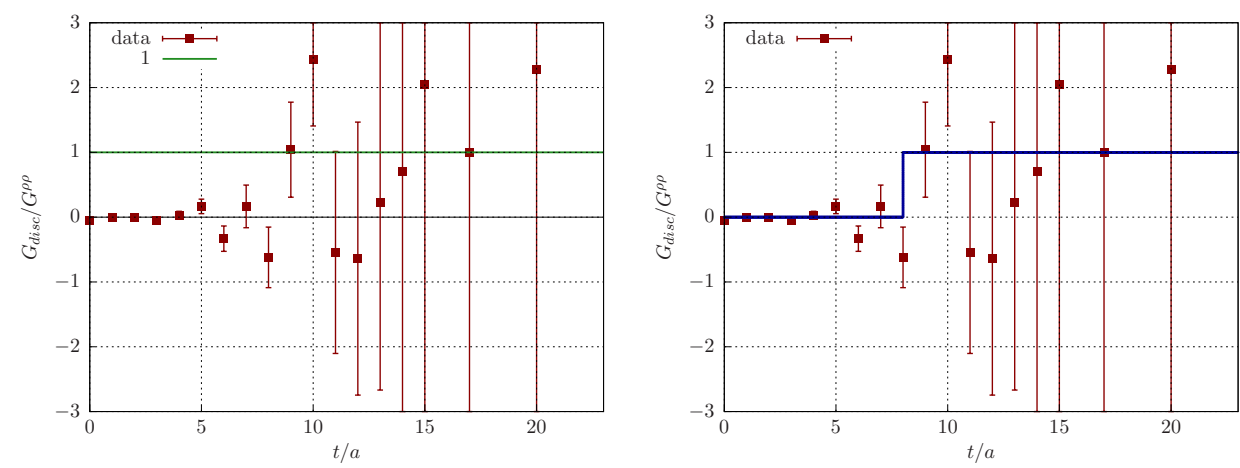

Figure 4: The ratio of the disconnected and the $\rho$-correlator. The green line one the left-hand side shows the asymptotic value. The blue curve on the right is our estimate for the maximum possible disconnected contribution.

\section{The $\gamma Z$ vacuum polarization with disconnected estimate}

To obtain an upper limit for the contribution of the disconnected diagram to the running of the Weinberg angle, we calculate the $\gamma Z$ vacuum polarization once without the disconnected contribution and once with an estimate for the disconnected contribution that follows the blue curve in figure 4. Both results are shown on the left-hand side of figure 5.
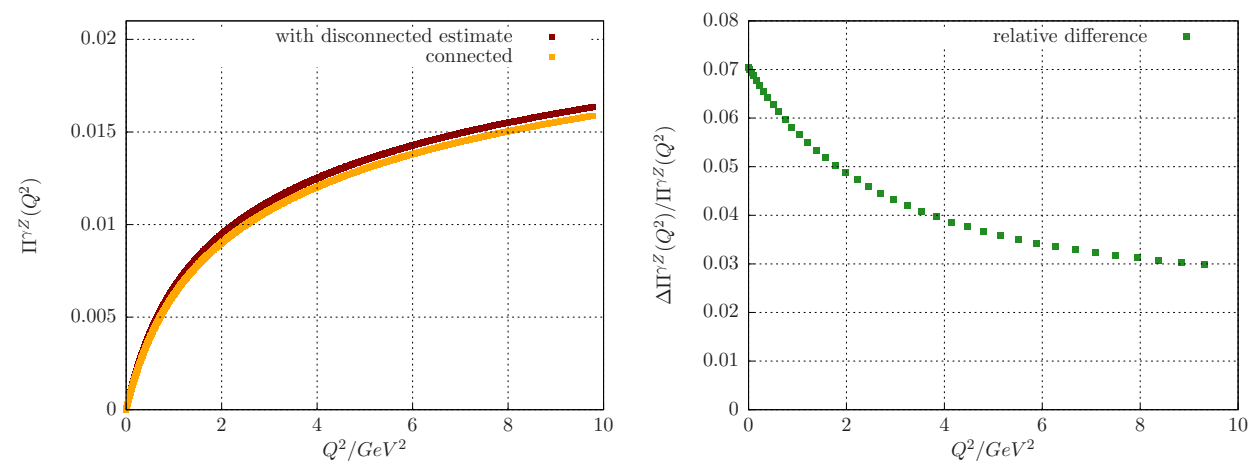

Figure 5: The $\gamma Z$ vacuum polarization once without disconnected contribution (yellow) and once with our estimate for the disconnected contribution (red). The green points on the right show the relative difference of these two curves.

We find that $\Pi_{R}^{\gamma Z}\left(Q^{2}\right)$ including the disconnected estimate is larger than the result from connected contributions only. Thus we can state, that the disconnected contribution to $\Pi_{R}^{\gamma Z}\left(Q^{2}\right)$ is positive. However, one has to keep in mind that our estimate only gives a conservative upper limit and the disconnected contribution might be much smaller. The right hand side of figure 5 shows the relative difference between the two curves at the left. One can see that at e.g. $Q^{2}=4 \mathrm{GeV}^{2}$ the upper limit for the disconnected contribution is $\approx 4 \%$ of the connected one. This $4 \%$ can be used as a systematic error that arises when the disconnected contribution is neglected.

Figure 6 shows the results for $\Delta_{\text {had }} \sin ^{2} \theta_{W}\left(Q^{2}\right)$ at $4 \mathrm{GeV}^{2}$ for three different pion masses (cf. table 1). Red points show the results from the connected contribution only. The asymmetric green error bar indicates the maximum possible disconnected contribution. On can see that the systematic error from the disconnected diagram is much larger than the statistical error on the connected result. Thus, our results for $\Delta_{\text {had }} \sin ^{2} \theta_{W}\left(Q^{2}\right)$ can only be improved with a better estimate of the 
disconnected contribution. The blue point in figure 6 is the result of a recent lattice calculation by Burger et al.[12], where disconnected contributions have been neglected.

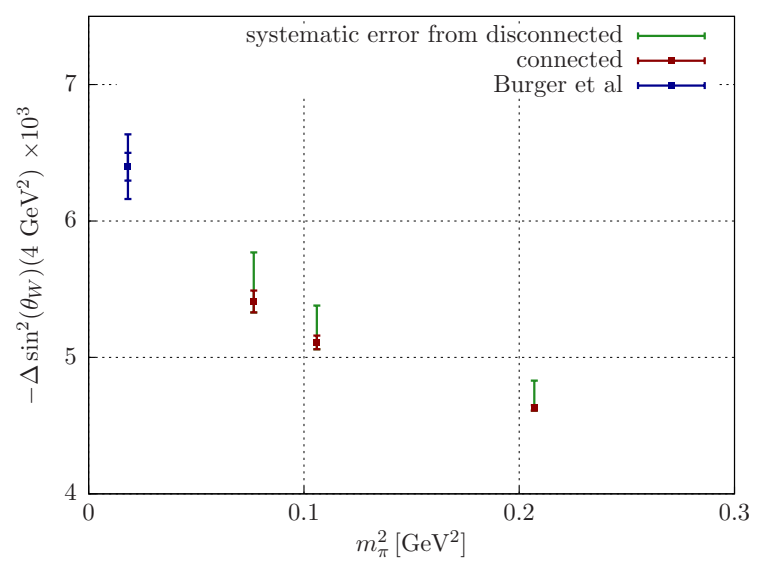

Figure 6: Our results for $\Delta_{\text {had }} \sin ^{2} \theta_{W}\left(Q^{2}\right)$ plotted against the pion mass. The blue point shows the result from Burger et al. [12].

Acknowledgements We acknowledge useful discussions with Benjamin Jäger, Anthony Francis and Gregorio Herdoíza. Our calculations were performed on the dedicated QCD platforms "Wilson" at the Institute for Nuclear Physics, University of Mainz, and "Clover" at the Helmholtz-Institut Mainz. We thank Dalibor Djukanovic and Christian Seiwerth for technical support. We are grateful for computer time allocated to project HMZ21 on the BlueGene computer "JUQUEEN" at NIC, Jülich. This research has been supported in part by the DFG in the SFB 1044. We are grateful to our colleagues in the CLS initiative for sharing ensembles.

\section{References}

[1] F. Jegerlehner, Z. Phys.C - Particles and Fields 32, 195-207 (1986)

[2] D. Bernecker and H. Meyer, Eur.Phys.J. A47 148 (2011), [arXiv:1107.4388]

[3] A. Francis, B. Jäger, H. Meyer and H. Wittig, Phys. Rev. D 88, 054502 (2013), [arXiv:1306.2532]

[4] X. Feng et al., Phys. Rev. D 88, 034505 (2013), [arXiv:1305.5878]

[5] F. Jegerlehner, Nuovo Cim. C034S1, 31-40 (2011), [arXiv:1107.4683]

[6] A. Francis, V. Gülpers, G. Herdoíza, H. Horch, B. Jäger, H. Meyer and H. Wittig, PoS LAT2014, (2014) 127, [arXiv:1410.7491]

[7] M. Della Morte et al., JHEP 0507, 007 (2005), [arXiv:hep-lat/0505026]

[8] T. Kinoshita et al., Phys.Rev.Lett. 109, 111807 (2012), [arXiv:1205.5368]

[9] K.S. Kumar et al., Ann.Rev.Nucl.Part.Sci. 63, 237-267 (2013), [arXiv:1302.6263]

[10] A. Francis, V. Gülpers, B. Jäger, H. Meyer, G. von Hippel and H. Wittig, PoS LAT2014, (2014) 128, [arXiv:1411.7592]

[11] V. Gülpers, G. von Hippel and H. Wittig, Phys. Rev. D 89, 094503 (2014), [arXiv:1309.2104]

[12] F. Burger, K. Jansen, M. Petschlies, G. Pientka, [arXiv:1505.03283] 